\title{
Variation in the Distribution of
} Putative Virulence and Colonization Factors in Shiga Toxin-Producing Escherichia coli Isolated from Different Categories of Cattle

\author{
Maria E. Cáceres ${ }^{1}$, Analía l. Etcheverria ${ }^{1 *}$, Daniel Fernández ${ }^{2}$, Edgardo M. Rodríguez ${ }^{3}$ and \\ Nora L. Padola ${ }^{1}$

\begin{abstract}
${ }^{1}$ Laboratorio de Inmunoquímica y Biotecnología, Departamento de Sanidad Animal y Medicina Preventiva, Centro de Investigación Veterinaria Tandil, CONICET, CICPBA, Tandil, Argentina, ${ }^{2}$ Laboratorio de Inmunoquímica y Biotecnología, Departamento de Sanidad Animal y Medicina Preventiva, Facultad de Ciencias Veterinarias, Tandil, Argentina, ${ }^{3}$ Área de Bioestadística, Departamento Sanidad Animal y Medicina Preventiva, Centro de Investigación Veterinaria, Facultad de

Ciencias Veterinarias, Tandil, Argentina
\end{abstract}

\section{OPEN ACCESS}

Edited by:

Alfredo G. Torres,

University of Texas Medical Branch,

USA

Reviewed by:

Séamus Fanning,

University College Dublin, Ireland

Grzegorz Wegrzyn,

University of Gdańsk, Poland

*Correspondence:

Analía I. Etcheverría

analiain@vet.unicen.edu.ar

Received: 22 February 2017 Accepted: 10 April 2017 Published: 28 April 2017

Citation:

Cáceres ME, Etcheverría Al, Fernández D, Rodríguez EM and Padola NL (2017) Variation in the Distribution of Putative Virulence and

Colonization Factors in Shiga

Toxin-Producing Escherichia coli Isolated from Different Categories of Cattle.

Front. Cell. Infect. Microbiol. 7:147. doi: 10.3389/fcimb.2017.00147
Shiga toxin-producing Escherichia coli (STEC) are pathogens of significant public health concern. Several studies have confirmed that cattle are the main reservoir of STEC in Argentina and other countries. Although Shiga toxins represent the primary virulence factors of STEC, the adherence and colonization of the gut are also important in the pathogenesis of the bacteria. The aim of this study was to analyze and to compare the presence of putative virulence factors codified in plasmid -katP, espP, subA, stcE-and adhesins involved in colonization of cattle -efa1, iha- in 255 native STEC strains isolated from different categories of cattle from different production systems. The most prevalent gene in all strains was espP, and the less prevalent was stcE. katP was highly detected in strains isolated from young and rearing calves (33.3\%), while subA was predominant in those isolated from adults $(71.21 \%)$. Strains from young calves showed the highest percentage of efa1 $(72.46 \%)$, while iha showed a high distribution in strains from rearing calves and adults (87.04 and $98.48 \%$ respectively). It was observed that espP and iha were widely distributed throughout all strains, whereas katP, stcE, and efa1 were more associated with the presence of eae and subA with the eae-negative strains. A great proportion of eae-negative strains were isolated from adults -dairy and grazing farms- and from rearing calves -dairy and feedlot-, while mostly of the eae-positive strains were isolated from dairy young calves. Data exposed indicate a correlation between the category of the animal and the production systems with the presence or absence of several genes implicated in adherence and virulence of STEC.

Keywords: shiga toxin-producing Escherichia coli, category of cattle, plasmid genes, adhesins, production systems 


\section{INTRODUCTION}

Shiga toxin-producing Escherichia coli (STEC) are endemic pathogens in Argentina with a high impact on the health system. STEC has been associated with outbreaks and sporadic cases of human disease, causing diarrhea, hemorrhagic colitis (HC) and hemolytic uremic syndrome (HUS) (Paton and Paton, 1998).

The primary virulence factors of STEC are Shiga toxins (stx), however the adherence and colonization of gut are also important and constitute the first step in the pathogenesis of the bacteria (Szalo et al., 2002). Another typical virulence factor is Intimin, an outer membrane adherence protein encoded by eae, located in the locus of enterocyte effacement (LEE), which is responsible for the histopathological lesion called "attaching and effacing" (A/E) (Nataro and Kaper, 1998). The LEE-pathogenicity island is present in a set of STEC serotypes considered to be highly virulent, however, LEE not appear to be essential for pathogenesis, since a large number of LEE-negative strains has been associated with sporadic outbreaks of HC and HUS (Cergole-Novella et al., 2007; Cundon et al., 2015). Other colonization factors beyond Intimin have been described: An adherence-conferring protein similar to the Vibrio cholerae IrgA (iha) that confer the capacity to adhere to epithelial cells in a diffuse pattern (Tarr et al., 2000) and an enterohemorrhagic E. coli factor of adherence (efal) implicated in the intestinal colonization of calves (Nicholls et al., 2000; Stevens et al., 2002).

There are additional putative virulence factors encoded by plasmids that contribute to the survival and citotoxicity of STEC, such as an hemolysin (ehxA) encoded by the plasmid pO157 with cytotoxic activity in human and bovine cell lines (Schmidt et al., 1995), a katalase-peroxidase (katP) which is part of bacterial defense mechanisms against oxidative stress, an extracellular serine protease $(\operatorname{esp} P)$ that is able to cleave pepsin and coagulation factor $\mathrm{V}$, suggesting that it might be an accessory virulence factor exacerbating HC (Caprioli et al., 2005; Brockmeyer et al., 2007), and a zink metalloprotease ( $s t c E)$, which modify the protective layer of the host intestine to assist the pedestal-actine formation encoded by LEE contributing to intimate adherence to cells (Grys et al., 2005). The presence of other toxins in addition to Stx has been reported, such as the subtilase cytotoxin $(s u b A B)$ encoded by the plasmid pO113 particulary carried by eae-negative STEC strains. This toxin is lethal for mice, inhibit protein synthesis, have pro-inflammatory properties, and may play a significant role in pathogenesis of STEC disease (Paton and Paton, 2010).

Several studies have confirmed that cattle are the main reservoir of STEC O157 and non-O157 serotypes in Argentina and other countries, with variable prevalence ranged from 22 to $62.7 \%$ in different categories of cattle (Sanz et al., 1998; Blanco et al., 2004; Padola et al., 2004). In young cattle, a prevalence of STEC was showed in 25,43 , and $58 \%$ in newborn calves, milkfed, and growing dairy calves, respectively. The presence of STEC in newborn calves $<24$ h old suggested that they are exposed to this bacterium quickly after birth, which migth play an important role in vertical STEC transmission (Fernández et al., 2012). Also, a high prevalence of STEC in dairy cows, preslaughter and healthy grazing cattle $-37.5,44$, and $22 \%$, respectively- has been detected (Sanz et al., 1998; Fernández et al., 2009). Recent studies confirm the age of cattle as an important factor that significantly influences the shed of STEC, more than other physiological factors, such as breed, sex or weight gain. Data revealed that calves were more likely to shed STEC during the first 6 months, peaked at 2 years of age and declines as the animal matured. It was found that diversity of gut microflora could be a reason for that variation (Mir et al., 2014, 2016).

Contact with feces of cattle, direct contact with the animals or their environment and consumption of contaminated beef, milk, dairy products, water, unpasteurized apple juices, and vegetables, are possible routes for STEC human exposure and disease (Cody et al., 1999; Olsen et al., 2002; Jure et al., 2010; Brusa et al., 2013). Because the most of the STEC human infections are caused by consumption of contaminated animal products, the knowledge of the virulence profiles and colonization factors of STEC among different animals categories could provides information to evaluate some strategies to control cattle shedding in order to decrease the incidence of disease in humans. Therefore, the aim of this study was to analyze and to compare the presence of putative virulence factors codified in megaplasmid and adhesins involved in colonization of cattle in a collection of STEC strains

TABLE 1 | Characteristics of the PCR primers used in this study.

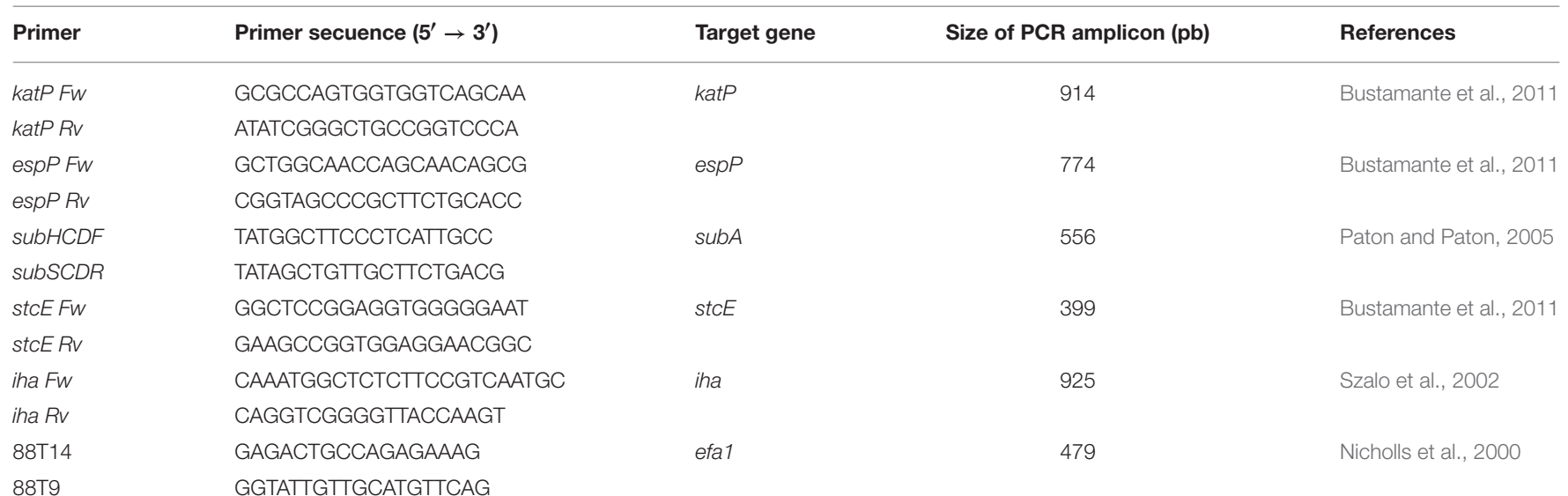


isolated from different cattle categories and different production systems of Argentina.

\section{MATERIALS AND METHODS}

\section{Bacterial Isolates}

A total of 255 eh $x A$-positive STEC strains from the Laboratorio de Inmunoquímica y Biotecnología (UNCPBA, Tandil, Buenos Aires, Argentina) were analyzed. All STEC strains were previously isolated from fecal samples of cattle from different categories, named in this study as: young calves (0-2 mo), rearing calves $(2-8 \mathrm{mo})$ and adults $(>8 \mathrm{mo})$, which came from different regional production systems (dairy farm, feedlot and grazing). STEC strains were serotyped and characterized for detection of typical virulence factors -stx1, stx2, eae, ehxA- and saa (Sanz et al., 1998; Padola et al., 2004; Fernández et al., 2010, 2012).

\section{PCR}

For the detection of megaplasmid genes -katP, espP, subA, stcE- a multiplex PCR was performed according to the methodology described by Bustamante et al. (2011). Two PCRs were performed for the detection of efal and iha according to that described by Nicholls et al. (2000) and Szalo et al. (2002), with some modifications. Primers and size of PCR products are shown in Table 1. PCR products were visualized by agarose gel (2\%) electrophoresis, with ethidium bromide staining.

\section{Statistical Analysis}

In order to describe the distribution of plasmid genes and adhesins between different categories of cattle, data were analyzed using the Chi2 test, or Fisher's exact test if necessary and possible, using the PROC FREQ procedure of Statistical Analysis Systems, Version 9.3 (SAS Institute, Cary, NY).

\section{RESULTS}

\section{Distribution of Plasmid and Adhesins Genes in STEC According the Category of Cattle}

The most prevalent gene detected among all strains regardless the category of cattle was espP, while $s t c E$ was the less prevalent ( $p=0.0679$ and $p=0.2670$, respectively). For katP, the differences observed between categories, young calves, rearing calves and adults $(33.3,33.3$, and $6.1 \%$, respectively) were statistically significant $(p<0.0001)$. SubA was mostly found in strains isolated from adults (71.21\%; $p<0.0001$; Figure 1).

Significant differences in the distribution of efal were observed, with a greater percentage in strains from young calves (72.46\%) than rearing calves $(29.63 \%)$ and adults $(6.06 \%)(p<$ 0.0001 ). The ditribution of iha showed the highest proportion in strains isolated from adults $(98.48 \% ; p=0.0126$; Figure 1).

The most prevalent virulence profile in STEC strains isolated from young calves was espP, efa1, iha (21.73\%), while espP, subA, iha was the most prevalent in rearing calves and adults (37 and $68.3 \%$ respectively) (Table 2 ).

\section{Comparison of Genes Distribution among Strains According Presence or Ausence of eae}

Most eae-positive strains were isolated from young calves from dairy farm and rearing calves from feedlot; while eae-negative serotypes were isolated mostly from dairy and grazing adults. $k a t P$, efal, and $s t c E$ were predominant in eae-positive serotypes while subA and iha were prevalent in eae-negative serotypes (Table 3).

\section{DISCUSSION}

Since that cattle has been recognized as the main reservoir of STEC, several strains from different sources and production systems have been isolated by our research group (Sanz et al.,

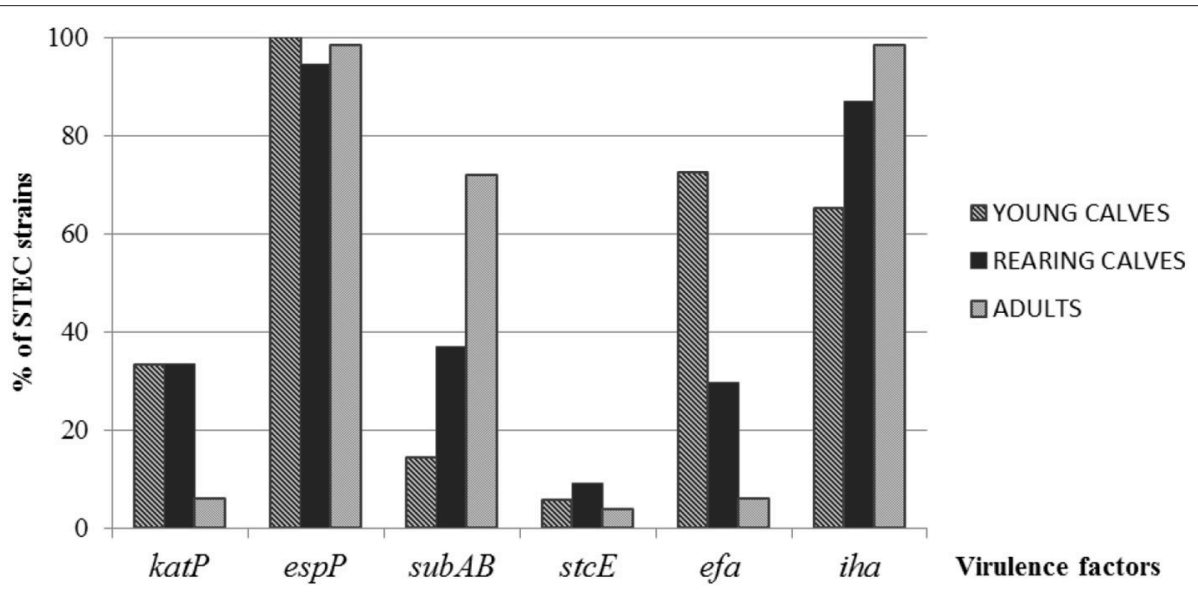

FIGURE 1 | Distribution of virulence factors in STEC strains among the different categories of cattle. 
TABLE 2 | Virulence profile carried by STEC clasified according to the category of cattle.

\begin{tabular}{|c|c|c|}
\hline Category of cattle & Virulence profiles & No. (\%) strains \\
\hline \multirow[t]{18}{*}{ Young calves $(n=69)$} & espP, efa1, iha & $15(21.73)$ \\
\hline & katP, espP, efa1, iha & $10(14.5)$ \\
\hline & espP, efa1 & $10(14.5)$ \\
\hline & katP, espP, efa1 & $8(11.6)$ \\
\hline & espP, subA, iha & $5(7.2)$ \\
\hline & espP, iha & 4 \\
\hline & espP, iha & 2 \\
\hline & katP, espP, stcE, efa1, iha & 2 \\
\hline & katP, espP, iha & 2 \\
\hline & espP, subA, iha & 2 \\
\hline & espP & 2 \\
\hline & katP, espP, efa1 & 1 \\
\hline & espP, subA, efa1, iha & 1 \\
\hline & espP, subA, efa1 & 1 \\
\hline & espP, subA, & 1 \\
\hline & espP, stcE, efa1, iha & 1 \\
\hline & espP, stcE & 1 \\
\hline & espP, efa1 & 1 \\
\hline \multirow[t]{14}{*}{ Rearing calves $(n=54)$} & espP, subA, iha & $20(37)$ \\
\hline & katP, espP, efa1, iha & $6(11)$ \\
\hline & katP, espP, iha & $5(9.2)$ \\
\hline & espP & $5(9.2)$ \\
\hline & espP, iha & $4(7.4)$ \\
\hline & katP, espP, stcE, efa1, iha & 3 \\
\hline & espP, efa1, iha & 2 \\
\hline & katP, espP, stcE, iha & 2 \\
\hline & efa1, iha & 2 \\
\hline & katP, espP, stcE, efa1 & 1 \\
\hline & espP, iha & 1 \\
\hline & katP, espP, efa1, iha & 1 \\
\hline & espP, ehxA, efa1 & 1 \\
\hline & iha & 1 \\
\hline \multirow[t]{11}{*}{ Adults $(n=132)$} & espP, subA, iha & $91(68.3)$ \\
\hline & espP, iha & $25(19)$ \\
\hline & katP, espP, stcE, efa1, iha & $3(2.2)$ \\
\hline & katP, espP, efa1, iha & 2 \\
\hline & katP, espP, iha & 2 \\
\hline & espP, subA, efa1, iha & 2 \\
\hline & espP, subA & 2 \\
\hline & iha & 2 \\
\hline & espP, efa1, iha & 1 \\
\hline & katP, espP, stcE, iha & 1 \\
\hline & espP, stcE, iha & 1 \\
\hline
\end{tabular}

1998; Parma et al., 2000; Padola et al., 2004; Fernández et al., $2009,2010,2012)$. The isolation and characterization of STEC from cattle is essential for the development of diagnostic and control tools to avoid the transmission of STEC to humans through the consumption of bovine derived contaminated food.
In recent studies, the prevalence of STEC in beef cattle were investigated and a strong correlation between age and shedding was found (Mir et al., 2014). Calves have been identified as the major excretors of STEC decreasing with the growth of the animal. Similarly, the presence of stx has been correlated to age, specially stx2, being detected in young calves. Genotyping of additional factors in STEC isolated from animals with different ages is necessary for a more appropriate knowledge of their risk for human health (Mir et al., 2016).

In this study, the age as a factor of significant influence in the distribution of some STEC virulence and colonization factors was demonstrated. In agreement with Bustamante et al. (2011) we found a higher prevalence of espP among all STEC strains regardless the category of cattle and the production system, and $s t c E$ was the less prevalent. While espP was widely detected among eae-positives and eae-negatives serotypes, stcE was detected in a few eae-positive serotypes, such as O157:H7, O64:NM and NT. To our knowledge, this is the first report of the presence of $s t c E$ in O64:NM. A higher prevalence of katP in STEC isolated from young and rearing calves was found. All of the kat $P$-positive strains also contained espP, and some of them harbored $s t c E$.

Subtilase cytotoxin (SubAB) was firstly described in STEC O113:H21 responsible of an outbreak of HUS (Paton et al., 1999). Then, subA gene was indentified in various eae-negative STEC serotypes isolated from cattle, food and human patients (Cergole-Novella et al., 2007; Galli et al., 2010; Wu et al., 2010). In this study, eae-negative STEC serotypes O113:H21, O116:H21, O130:H11, O178:H19, and ONT:H7 carried subA and were isolated mostly from rearing calves and adults from dairy and grazing systems and, in agreement with Bustamante et al. (2011), none of $s u b A$-positive STEC harbored neither $s t c E$ nor katP.

Some putative adhesins have been described to inquire the mechanisms underlying the adherence of STEC strains to epithelial cells. In this study, wide differences between cattle categories were found regarding the adhesins investigated. In those STEC strains isolated from dairy young calves and feedlot rearing calves, high percentages of efa 1 were detected, declining with the increasing of age. In agreement with previous reports (Nicholls et al., 2000; Toma et al., 2004; Galli et al., 2010) the $89.5 \%$ of the efal-positive strains were also eae-positive. Several authors have detected a high prevalence of iha in STEC strains isolated from animals, food, and humans, most of them eae-negative (Cergole-Novella et al., 2007; Galli et al., 2010). Our results show that iha was detected in all of STEC strains regarding the category of cattle, although it was more prevalent in those isolated from adults, and in eae-negatives STEC serotypes. However, Kobayashi et al. (2013) found high percentages of iha in eae-positives STEC strains, suggesting that this gene could have a wide distribution in STEC beyond the presence of eae.

Differences in genetic characteristics of STEC strains in reference to age may be explained by gut diversity microflora composition, low concentration of normal Enterobacteriaceae microflora that should influence STEC survival, adherence and colonization in the cattle intestine (Zhao et al., 2013). 
TABLE 3 | Virulence profiles in STEC strain according to presence or absence of eae.

\begin{tabular}{|c|c|c|c|c|c|}
\hline \multicolumn{3}{|c|}{ eae $+(n=89)$} & \multicolumn{3}{|c|}{ eae $-(n=166)$} \\
\hline Virulence profiles & No. of strains & Serotypes & Virulence profiles & No. of strains & Serotypes \\
\hline espP, efa1, iha & 18 & \multirow{16}{*}{$\begin{array}{c}\text { O5:NM, O8:H16, O8:H25, } \\
\text { O20:HNT, O26:H11, O38:H39, } \\
\text { O103:NM, O103:H2/H8/H18, } \\
\text { O111:NM, O113:H2, O118:H16, } \\
\text { O145:NM, O146:NM, O153:H25, } \\
\text { O157:H7, O165:NM, O171:H25, } \\
\text { O172:NM/H21, NT }\end{array}$} & espP, subA, iha & 116 & \multirow{16}{*}{$\begin{array}{c}\text { O2:H5, O8:H19/H20, O20:H19, } \\
\text { O27:H21, O37:H10, O39:H49, } \\
\text { O46:H11, O41:H38, O55:NM, } \\
\text { O64:NM, O74:H28, O79:NM, } \\
\text { O88:H25, O91:H8/H21/H28, } \\
\text { O103:H21, O105:H18, } \\
\text { O113:H2/H21, O116:H21, } \\
\text { O130:H11, O141:H7/H8, } \\
\text { O153:H21/H25, O163:H19/H21, } \\
\text { O174: H21, O178:H2/H7/H8/ } \\
\text { H19/H25/H28/H29, O179:NM, } \\
\text { ONT:H7/H11/H46, NT, } \\
\text { Autoaglutinante. }\end{array}$} \\
\hline katP, espP, efa1, iha & 18 & & espP, iha & 31 & \\
\hline espP, efa1 & 10 & & espP & 5 & \\
\hline katP, espP, efa1 & 9 & & iha & 2 & \\
\hline katP, espP, stcE, efa1, iha & 8 & & espP, subA & 2 & \\
\hline katP, espP, iha & 7 & & espP, subA, efa1, iha & 2 & \\
\hline espP, iha & 5 & & katP, espP, iha & 2 & \\
\hline espP & 2 & & espP, efa1 & 1 & \\
\hline espP, subA, iha & 2 & & espP, stcE, iha & 1 & \\
\hline katP, espP, stcE, iha & 2 & & katP, espP, stcE, iha & 1 & \\
\hline efa1, iha & 2 & & katP, espP, efa1 & 1 & \\
\hline espP, subA & 1 & & katP, espP, efa1, iha & 1 & \\
\hline espP, subA, efa1 & 1 & & & & \\
\hline espP, subA, efa1, iha & 1 & & & & \\
\hline espP, stcE & 1 & & & & \\
\hline iha & 1 & & & & \\
\hline
\end{tabular}

In adults animals, prevalence of STEC decrease while the commensal microflora increase its diversity, possibly influenced by diet of the animals which changes as the animal weans, grows and begins to incorpore pasture, or specific diet according the production system (Mir et al., 2016).

Data exposed indicate a correlation between the category of the animal and the production systems with the presence or absence of several genes implicated in adherence and virulence of STEC. Further studies are necessary to better understand the involvement of these genes in the dynamic of colonization and survival of this pathogen in cattle in order to establish control strategies to reduce the transmission of STEC from animals to humans.

\section{REFERENCES}

Blanco, M., Blanco, J. E., Mora, A., Dahbi, G., Alonso, M. P., González, E. A., et al. (2004). Serotypes, virulence genes, and intimin types of Shiga toxin (verotoxin)-producing Escherichia coli isolates from cattle in Spain and identification of a new intimin variant gene (eae- $\xi$ ). J. Clin. Microbiol. 42, 645-651. doi: 10.1128/JCM.42.2.645-651.2004

Brockmeyer, J., Bielaszewska, M., Fruth, A., Bonn, M. L., Mellmann, A., Humpf, H.-U., et al. (2007). Subtypes of the plasmid-encoded serine protease EspP in shiga toxin-producing Escherichia coli: distribution, secretion, and proteolytic activity. Appl. Environ. Microbiol. 73, 6351-6359. doi: 10.1128/AEM.00920-07

Brusa, V., Aliverti, V., Aliverti, F., Ortega, E. E., de la Torre, J. H., Linares, L. H., et al. (2013). Shiga toxin-producing Escherichia coli in beef retail markets from Argentina. Front. Cell. Infect. Microbiol. 2:171. doi: 10.3389/fcimb.2012.00171

Bustamante, A. V., Sanso, A. M., Lucchesi, P. M. A., and Parma, A. E. (2011). Multiplex PCR assay for the detection of five putative virulence gene encoded in verotoxigenic Escherichia coli plasmids. Curr. Microbiol. 62, 1411-1415. doi: 10.1007/s00284-011-9877-5

Caprioli, A., Morabito, S., Brugère, H., and Oswald, E. (2005). Enterohaemorragic Escherichia coli: emerging issues on virulence and modes of transmission. Vet. Res. 36, 289-311. doi: 10.1051/vetres:2005002

\section{AUTHOR CONTRIBUTIONS}

MC designed and performed the experiments, analyzed the data, wrote the paper. DF performed the experiments, revised the manuscript. ER analyzed statistically the data. AE and NP designed the experiments and revised critically the manuscript.

\section{ACKNOWLEDGMENTS}

Authors thank María R. Ortiz, from the Laboratorio de Inmunoquímica y Biotecnología (UNCPBA, Tandil, Buenos Aires, Argentina) for her technical assistance. This work was supported by PICT 2015/2666 and PICT 2013/1749 y SECAT, UNCPBA.

Cergole-Novella, M. C., Nishimura, L. S., Fernando dos Santos, L., Irino, K., Vaz, T. M., Bergamini, A. M., et al. (2007). Distribution of virulence profiles related to new toxins and putative adhesins in Shiga toxin-producing Escherichia coli isolated from diverse sources in Brazil. FEMS Microbiol. Lett. 274, 329-334. doi: 10.1111/j.1574-6968.2007.00856.x

Cody, S. H., Glynn, M. N., Farrar, J. A., Cairns, K. L., Griffin, P. M., Kobayashi, J., et al. (1999). An outbreak of Escherichia coli O157:H7 infection from unpasteurized commercial apple juice. Ann. Intern. Med. 130, 202-209. doi: 10.7326/0003-4819-130-3-199902020-00005

Cundon, C., Marey, E., Roldán, F., Canosa Montero, C. S., Navarro, A., Padola, N. L., et al. (2015). Detección y Caracterización Preliminar de Escherichia coli O174 Productor de Toxina. Buenos Aires: SNS Publicación periodística científico-tecnológica, 2314-2901.

Fernández, D., Irino, K., Sanz, M. E., Padola, N. L., and Parma, A. E. (2010). Characterization of Shiga toxin-producing Escherichia coli isolated from dairy cows in Argentina. Lett. App. Microbiol. 51, 377-382. doi: 10.1111/j.1472-765X.2010.02904.x

Fernández, D., Rodríguez, E. M., Arroyo, G., Padola, N. L., and Parma, A. E. (2009). Seasonal variation of Shiga toxin-encoding genes (stx) and detection of E. coli $\mathrm{O} 157$ in dairy cattle from Argentina. J. App. Microbiol. 106, 1260-1267. doi: 10.1111/j.1365-2672.2008.04088.x 
Fernández, D., Sanz, M. E., Parma, A. E., and Padola, N. L. (2012). Short communication: characterization of Shiga toxin-producing Escherichia coli isolated from newborn, milk-fed, and growing calves in Argentina. J. Dairy Sci. 95, 5340-5343. doi: 10.3168/jds.2011-5140

Galli, L., Miliwebsky, E., Irino, K., Leotta, G., and Rivas, M. (2010). Virulence profile comparison between LEE-negative Shiga toxin-producing Escherichia coli (STEC) strains isolated from cattle and humans. Vet. Microbiol. 143, 307-313. doi: 10.1016/j.vetmic.2009.11.028

Grys, T. E., Siegel, M. B., Lathem, W. W., and Welch, R. A. (2005). The StcE protease contributes to intimate adherence of enterohemorrahic Escherichia coli O157:H7 to host cells. Infect. Inmun. 73, 1295-1303. doi: 10.1128/IAI.73.3.1295-1303.2005

Jure, M. A., Condorí, S., Leotta, G. A., Chinen, I., Miliwebsky, E., Allori, C., et al. (2010). Detección, aislamiento y caracterización de Escherichia coli productor de toxina Shiga a partir de carne molida fresca proveniente de carnicerías de Concepción, provincia de Tucumán. Rev. Argent. Microbiol. 42, 284-287. doi: 10.1590/S0325-75412010000400009

Kobayashi, N., Lee, K., Yamazaki, A., Saito, S., Furukawa, I., Kono, T., et al. (2013). Virulence gene profiles and population genetic analysis for exploration of pathogenic serogroups of Shiga toxin-producing Escherichia coli. J. Clin. Microbiol. 51, 4022-4028. doi: 10.1128/JCM.01598-13

Mir, R. A., Weppelmann, T. A., Elzo, M., Ahn, S., Driver, J. D., and Jeong, K. C. (2016). Colonization of beef cattle by Shiga toxin-producing Escherichia coli during the first year of life: a cohort study. PLoS ONE 11:e0148518. doi: 10.1371/journal.pone.0148518

Mir, R. A., Weppelmann, T. A., Kanga, M., Bliss, T. M., DiLorenzo, N., Lamb, G. C., et al. (2014). Association between animal age and the prevalence of Shiga toxin-producing Escherichia coli in a cohort of beef cattle. Vet. Microbiol. 175, 325-331. doi: 10.1016/j.vetmic.2014.12.016

Nataro, J. P., and Kaper, J. B. (1998). Diarrheagenic Escherichia coli. Clin. Microbiol. Rev. 11, 142-201.

Nicholls, L., Grant, T. H., and Robins-Browne, R. M. (2000). Identification of a novel genetic locus that is required for in vitro adhesion of a clinical isolate of enterohaemorragic Escherichia coli to epithelials cells. Mol. Microbiol. 35, 275-288. doi: 10.1046/j.1365-2958.2000.01690.x

Olsen, S. J., Miller, G., Breuer, T., Kennedy, M., Higgins, C., Walford, J., et al. (2002). A waterborne outbreak of Escherichia coli O157:H7 infections and hemolytic uremic syndrome: implications for rural water systems. Emerg. Infect. Dis. 8, 370-375. doi: 10.3201/eid0804.000218

Padola, N. L., Sanz, M. E., Blanco, J. E., Blanco, M., Blanco, J., Etcheverría, A. I., et al. (2004). Serotypes and virulence genes of shigatoxigenic Escherichia coli (STEC) isolates from a feedlot in Argentina. Vet. Microbiol. 100, 3-9. doi: 10.1016/S0378-1135(03)00127-5

Parma, A. E., Sanz, M. E., Blanco, J. E., Blanco, J., Vi-as, M. R., Blanco, M., et al. (2000). Virulence genotypes and serotypes of verotoxigenic Escherichia coli isolated from cattle and foods in Argentina. Importance in public health. Eur. J. Epidemiol. 16, 757-762. doi: 10.1023/A:1026746016896

Paton, A. W., and Paton, J. C. (2005). Multiplex PCR for direct detection of shiga toxigenic Escherichia coli strains producing the novel subtilase cytotoxin. J. of Clin. Microbiol. 43, 2944-2947. doi: 10.1128/JCM.43.6.29442947.2005
Paton, A. W., and Paton, J. C. (2010). Escherichia coli Subtilase cytotoxin. Toxins (Basel). 2, 215-228. doi: 10.3390/toxins2020215

Paton, A. W., Woodrow, M. C., Doyle, R. M., Lanser, J. A., and Paton, J. C. (1999). Molecular characterization of a Shiga-toxigenic Escherichia coli O113:H21 strain lacking eae responsible for a cluster of cases of hemolytic-uremic syndrome. J. Clin. Microbiol. 37, 3357-3361.

Paton, J. C., and Paton, A. W. (1998). Pathogenesis and diagnosis of Shiga toxin-producing Escherichia coli infections. Clin. Microbiol. Rev. 11, 450-479.

Sanz, M. E., Vi-as, R. M., and Parma, A. E. (1998). Prevalence of bovine verotoxinproducing Escherichia coli in Argentina. Eur. J. Epidemiol. 14, 399-403. doi: 10.1023/A:1007427925583

Schmidt, H., Beutin, L., and Karch, H. (1995). Molecular analysis of the plasmidencoded hemolysin of Escherichia coli O157:H7 strain EDL933. Infect. Inmun $63,1055-1061$.

Stevens, M. P., van Diemen, P. M., Frankel, G., Philips, A. D., and Wallis, T. S. (2002). Efal Influences colonization of the bovine intestine by Shiga toxin-producing Escherichia coli serotypes O5 and O111. Infect. Immun. 70, 5158-5166. doi: 10.1128/IAI.70.9.5158-5166.2002

Szalo, I. M., Goffaux, F., Pirson, V., Piérard, D., Ball, H., and Mainil, J. (2002). Presence in bovine enteropathogenic (EPEC) and enterohaemorrhagic (EHEC) Escherichia coli of genes encoding for putative adhesins of human EHEC strains. Res. Microbiol. 153, 653-658. doi: 10.1016/S0923-2508(02)01379-7

Tarr, P. I., Bilge, S. S., Vary, J. C. Jr., Jelacic, S., Habeeb, R. L., Ward, T. R., et al. (2000). Iha: a novel Escherichia coli O157:H7 adherence-conferring molecule encoded on a recently acquired chromosomal island of conserved structure. Infect. Immun. 68, 1400-1407. doi: 10.1128/IAI.68.3.1400-1407.2000

Toma, C., Martínez Espinosa, E., Song, T., Miliwebsky, E., Chinen, I., Iyoda, S., et al. (2004). Distribution of putative adhesins in Shiga toxin-producing Escherichia coli of different seropathotypes. J. Clin. Microbiol. 42, 4937-4946. doi: 10.1128/JCM.42.11.4937-4946.2004

Wu, Y., Hinenoya, A., Taguchi, T., Nagita, A., Shima, K., Tsukamoto, T., et al. (2010). Distribution of virulence genes related to adhesins and toxins in Shiga toxin-producing Escherichia coli strains isolated from healthy cattle and diarrheal patients in Japan. J. Vet. Med. Sci. 72, 589-597. doi: 10.1292/jvms.09-0557

Zhao, L., Tyler, P. J., Starnes, J., Bratcher, C. L., Rankins, D., McCaskey, T. A., et al. (2013). Correlation analysis of Shiga toxin-producing Escherichia coli shedding and faecal bacterial composition in beef cattle. J. Appl. Microbiol. 115, 591-603. doi: $10.1111 /$ jam. 12250

Conflict of Interest Statement: The authors declare that the research was conducted in the absence of any commercial or financial relationships that could be construed as a potential conflict of interest.

Copyright (c) 2017 Cáceres, Etcheverría, Fernández, Rodríguez and Padola. This is an open-access article distributed under the terms of the Creative Commons Attribution License (CC BY). The use, distribution or reproduction in other forums is permitted, provided the original author(s) or licensor are credited and that the original publication in this journal is cited, in accordance with accepted academic practice. No use, distribution or reproduction is permitted which does not comply with these terms. 